\title{
The relationship between vagal nerve activity and clinical outcomes in prostate and non-small cell lung cancer patients
}

\author{
MARIJKE DE COUCK ${ }^{1}$, DAVID VAN BRUMMELEN ${ }^{2}$, \\ DENIS SCHALLIER $^{2}$, JACQUES DE GRÈVE ${ }^{2}$ and YORI GIDRON ${ }^{1}$ \\ ${ }^{1}$ Faculty of Medicine and Pharmacy, Free University of Brussels (VUB); \\ ${ }^{2}$ Department of Medical Oncology, Oncological Center UZ Brussels, Brussels, Belgium
}

Received November 27, 2012; Accepted January 7, 2013

DOI: $10.3892 /$ or.2013.2725

\begin{abstract}
Recent studies suggest that vagal nerve activity, indexed by heart rate variability (HRV), could have a prognostic role in cancer. However, most studies did not control adequately for confounders and included cardiac patients. Furthermore, the validity of this prognostic role needs to be tested in different types of cancer. The present study tested the prognostic role of HRV in prostate cancer (PC) and non-small cell lung cancer (NSCLC) patients, using a historical prospective design. HRV was derived from brief $10 \mathrm{sec}$ ECGs obtained at approximately the time of diagnosis in $113 \mathrm{PC}$ patients and 133 NSCLC patients. Outcomes included prostate-specific antigen (PSA) at 6 and 24 months in PC, and overall survival (OS) (for the full sample) and survival time (for the deceased patients) in NSCLC. Furthermore, the possible mediating role of C-reactive protein (CRP) was tested (in NSCLC), as well as whether age and stage moderated the relationship between HRV and prognosis in both types of cancer. In the PC patients, HRV significantly inversely predicted PSA levels at 6 and 24 months, independent of confounders. Furthermore, this was particularly significant in metastatic PC patients, indicating moderation by stage. In NSCLC patients, HRV did not predict OS and survival time, but it did positively predict survival time in patients under the age of 65 , independent of confounders. Additionally, CRP was not found to mediate the relationship between HRV and OS or survival time in NSCLC. The present results partly support previous studies and extend them to two additional common types of cancer, using a more rigorous control over confounders. Together with recent experimental findings, these results propose a modulatory role of vagal nerve activity in cancer. Therefore, routine measurement of HRV in estimating prognosis in cancer may be considered.
\end{abstract}

Correspondence to: Dr Marijke De Couck, Faculty of Medicine and Pharmacy, Free University of Brussels (VUB), 103 Laarbeeklaan, 1090 Jette, Brussels, Belgium

E-mail: marijke.de.couck@vub.ac.be

Key words: heart rate variability, cancer prognosis, neuromodulation, predictive factor, early diagnosis

\section{Introduction}

The incidence of prostate cancer (PC) has increased in recent years with the promotion of the prostate-specific antigen (PSA) test and the increase in the growth of the elderly population (1). The most important clinical prognostic markers currently available for PC are the PSA serum level and TNM staging including the Gleason score. Numerous studies support the prognostic role of PSA, particularly the PSA doubling time (2). In advanced disease, some recent therapeutic progress has been made, such as the drug abiraterone (3). However, the long term outcome remains dismal and overall therapeutic options, particularly in castration and hormone resistant cancer, remain limited.

While PC is the most common male cancer, non-small cell lung cancer (NSCLC) is among the most fatal ones. The outcome following resection of early-stage NSCLC is poor, with $35-50 \%$ recurrence rate resulting in mortality (1). Little progress has been made in the past 30 years in the reduction of distant recurrence and subsequent mortality (1), which remain unacceptably high, even for patients with stage I disease in whom no nodal or other metastatic involvement can be detected at the time of surgery (4). Recent improvements in diagnosis and treatment have been made, but they only apply to a minority of lung cancer cases, particularly in non-smokers. Thus, both epidermal growth factor receptor (EGFR) mutations (5) and Alk gene rearrangements (6) have become important prognostic and predictive biomarkers. For most cases of lung cancer, TNM classification, age, C-reactive protein (CRP) and forcedexpiratory volume in one second (FEV1) significantly influence the choice of treatment and strongly predict patient overall survival (OS) (7-9). Due to the high unmet medical needs in both types of cancer, it is critical to identify new prognostic markers that may eventually also form the basis for novel therapeutic approaches.

Attention has recently focused on the vagus nerve, due to its role in three mechanisms essential for tumorigenesis and progression in cancer. The first mechanism is oxidative stress (10), which leads to both DNA-damage, a central trigger of tumorigenesis, and to uncontrolled cell proliferation (11). Furthermore, DNA damage and oxidative stress are also predictive factors of prognosis (12). The second process is local excessive inflammation promoting tumorigenesis in early 
stages (13), and disease progression in its later stages $(14,15)$. The inflammatory microenvironment, normally recruited to help fight/eliminate the tumors, also promotes tumor growth. Finally, the metastatic process is also under the control of sympathetic neurotransmitters, which influence the migratory ability of cancer cells and determine the direction and the development of metastases (16). Vagal nerve activity is inversely related to and inhibits all these three mechanisms (17-21). Based on converging evidence, Gidron et al (22) hypothesized that vagal nerve activity may modulate tumor growth, and if this model is valid, might become a variable that could be used as a prognostic factor and could be therapeutically manipulated.

The vagal nerve activity index, i.e., heart rate variability (HRV), represents the time differences between successive heartbeats (also known as the beat-to-beat intervals), and is synonymous with $R R$ variability. The $R$ wave refers to the $R$ waves on the electrocardiogram corresponding to ventricular depolarization (23). Analysis of the time differences between successive heart beats can be accomplished with reference to time (time domain analysis) or frequency (frequency domain analysis). In this study, we focused on the time domain analyses, i.e., standard deviation of normal beat to beat intervals (SDNN), in msec, and root mean square successive difference (RMSSD), in msec.

HRV is highly correlated with vagal nerve activity $(\mathrm{r}=0.88)(24)$ and is mainly under the control of and reflects efferent cardiac vagal nerve activity. High HRV has been shown to predict longer cancer survival time and reduced tumor burden (25-27). However, those studies had small sample sizes and did not control for any confounder. A subsequent study (28) showed that HRV was a significant predictor of survival time in terminal cancer patients, independent of confounders. However, that study did not control for type of cancer. These studies also included patients with cardiac diseases or other conditions known to affect HRV. We recently showed that low HRV predicts increases in the colon marker carcinoembryonic antigen (CEA), independent of confounders and excluding cardiac patients (29). However, the sample size of the latter study was also relatively small $(\mathrm{N}=72)$, and baseline levels of the tumor marker were not considered. Moreover, it remains unknown whether these findings may be generalized to other types of cancer, and what the underlying mechanisms may be. For this reason, we focused on two prognostic and symptomatically different types of cancer.

The purpose of the present study was to test the relationship between vagal nerve activity, indexed by HRV, and prognosis in patients with PC and NSCLC, two types of cancer of different severity. Furthermore, we tested whether inflammatory factors, such as C-reactive protein (CRP), mediate, i.e., explain, this relationship. Moreover, the predictors age and stage were explored preliminarily as possible modulators (moderators) of the HRV-prognosis relationship. These moderators were chosen since it was expected that the role of new prognostic factors may differ between age and stage. Age has been shown to be a major determinant in multiple physiological systems (30). Furthermore, vagal nerve activity may play different prognostic roles in different stages, due to the possible different roles of its underlying mechanisms in different stages. Additionally, by looking at multiple tumor marker outcomes (in PC), we sought to determine at which time point vagal nerve activity has prognostic value.

We hypothesized that lower HRV would predict increased tumor marker and reduced OS and survival time. It was also hypothesized that these relationships would be mediated by an inflammatory marker and might be moderated by stage and age.

\section{Materials and methods}

Patients. Following approval of the Medical Ethics Committee, UZ Brussels, medical records of 620 patients with PC and 650 patients with NSCLC, treated at UZ Brussels between January 2005 and December 2009, were reviewed. Patients diagnosed with NSCLC or PC, with an intact ECG near diagnosis and available data for PSA (PC) were included. Exclusion criteria included conditions known to alter HRV or influence inflammation, such as inflammatory diseases (such as, arthritis), cardiovascular disease, implanted pacemaker, or prescribed cardiologic medication ( $\beta$-blockers, anti-arrhythmic), anemia, spleen and thyroid diseases.

Initially, we included $620 \mathrm{PC}$ and 650 NSCLC patients, of whom 113 PC and 133 NSCLC patients met our inclusion criteria. Most patients were excluded due to the lack of ECG in their medical files. Based on the effect sizes previously observed with smaller samples $(27,29)$, the sample sizes in both PC and NSCLC were expected to be sufficient to detect statistically significant relationships and to explore for moderators.

Design. The study included a historical prospective design, formally known as a retrospective design. We collected archival electronic patient records (historical) and examined the prospective relationship between HRV measured at baseline (time of diagnosis, Time 1), and prognosis at Time 2 (e.g., PSA at 6 months, OS). This design is commonly used in the reanalysis of existing data sets $(31,32)$.

\section{Measures}

Confounders. Background information included patient age, gender (NSCLC), treatments (radiotherapy, chemotherapy, surgery, hormonal), body mass index (BMI), FEV1 (NSCLC), TNM staging in NSCLC including Gleason score (PC), smoking history in units of pack years (NSCLC), and baseline PSA (PC). Data on other confounders, such as family history, were absent in several cases.

Vagal nerve activity. This was measured by HRV, obtained from $10 \mathrm{sec}$ ECGs performed at diagnosis. The MUSE ${ }^{\circledR}$ cardiology information system in the UZ Brussels hospital provided the distances (in msec) between the RR peaks in each ECG, for determining HRV. Time domain HRV parameters, i.e., SDNN in msec, and RMSSD in msec, were derived. Power spectral analysis HRV parameters (such as HF and LF) require longer recording periods, and thus, were not measured. Such short ECGs have been found to correlate with ECGs of longer durations (5 and $10 \mathrm{~min})$, particularly with respect to $\operatorname{RMSSD}(33,34)$, but also for SDNN (24 h) (35). Furthermore, $10 \mathrm{sec}$ SDNN has been shown to predict tumor marker levels in colon cancer (29). For the moderation analyses, a cut-off of $20 \mathrm{msec}$ was used, as in previous studies in cancer (29), to distinguish between patients with high vs. low SDNN and RMSSD.

Inflammatory marker. This included CRP, only for NSCLC. 


\section{Outcomes}

Non-small cell lung cancer. OS (in the full sample) and duration of survival time in the deceased patients.

Prostate cancer. PSA in $\mathrm{ng} / \mathrm{ml}$ at 6 months and 2 years.

Statistical analysis. Univariate associations were tested between all background variables and all outcomes and the HRV parameters, using Pearson correlations for continuous variables and t-tests for categorical data. A partial correlation tested whether HRV still predicted survival time (in NSCLC) or tumor marker levels (in PC), after controlling for all significant background factors. When testing for moderation (interactions of HRV $\mathrm{x}$ age and HRV $\mathrm{x}$ stage), the cut-off of $20 \mathrm{msec}$ for SDNN and RMSSD to predict outcomes was used, independent of confounders. For the main analyses, we used a Bonferroni correction for both types of cancer. In the PC analyses, we had two measurements of HRV and two outcomes of PSA, resulting in 4 tests, requiring a significance level $\alpha$ of $0.05 / 4=0.0125$. In the lung cancer analyses, we had two measurements of HRV and two outcomes (OS and survival time), resulting in four tests, requiring a significance level $\alpha$ of $0.05 / 4=0.0125$. Since the moderation part is only an exploratory analysis, we did not use a corrected $\alpha$ for these additional tests.

\section{Results}

Prostate cancer. Of the 620 reviewed PC patients, only 113 met our inclusion criteria (for the majority of the excluded patients, no ECG or PSA levels were available). The descriptive statistics of the study variables are shown in Table I, however the continuous variables are not log transformed for purpose of clarity.

As shown in Table I, various treatments were used, and the percentages exceeded a $100 \%$ since a multi-modality therapeutic approach was used for several patients. Due to the lack of normal distributions, the scores of PSA, SDNN and RMSSD were $\log$ transformed, on which the following analyses were performed. Furthermore, this was carried out due to the large $\mathrm{SD}$, reflecting large inter-patient variability, in PSA levels. In the following analyses, for purposes of brevity, we used the terms SDNN, RMSSD, CRP and HRV, which refer to the log transformed measurements of these parameters.

The relationship between each measured confounder and both the predictor HRV (SDNN for this analysis), and PSA levels at 6 months, was tested. Among all confounders, stage (including Gleason score), radiotherapy, hormonal treatment, age and baseline PSA were significantly related to PSA levels at 6 months. Surgery was not related to either PSA or HRV. None of the confounders were correlated with SDNN. Concerning the confounders, in patients who did not receive radiotherapy or hormonal treatment, PSA levels at 6 months were significantly lower than in patients who received either treatment $(\mathrm{p}=0.018$ and $\mathrm{p}=0.02$, respectively). Age and baseline PSA were significantly positively correlated with PSA levels at 6 months $(r=0.414 ; \mathrm{p}=0.001$ and $\mathrm{r}=0.48 ; \mathrm{p}<0.001$, respectively); stage was also associated with PSA levels at 6 months $(\mathrm{p}=0.001)$.

With regard to vagal nerve activity and PSA, in univariate analyses, SDNN significantly inversely predicted PSA levels
Table I. Descriptive statistics of the prostate cancer sample.

A. Categorical variables

Variable

Radiotherapy

42.5

Hormonal therapy

Chemotherapy

Surgery

Stage

Stage 2

64.5

Stage 3

Stage 4

25.8

\section{B. Continuous variables}

\begin{tabular}{lc} 
Variable & $($ Mean \pm SD) \\
\hline Age (years) & $65.06 \pm 8.87$ \\
Heart rate & $73.32 \pm 14.00$ \\
HRV variables & \\
SDNN & $31.24 \pm 30.27$ \\
RMSSD & $32.34 \pm 40.25$ \\
PSA & \\
Baseline PSA & $130.00 \pm 520.67$ \\
PSA 6 months & $9.44 \pm 36.24$ \\
PSA 2 years & $2.84 \pm 10.50$
\end{tabular}

HRV, heart rate variability; SDNN, standard deviation of normal RR intervals; RMSSD, root mean square successive difference; PSA, prostate-specific antigen.

at 6 months $(\mathrm{r}=-0.399 ; \mathrm{p}=0.002)$. Furthermore, RMSSD also significantly inversely predicted PSA levels at 6 months $(\mathrm{r}=-0.345 ; \mathrm{p}=0.009)$ and tended to significantly predict PSA levels at 2 years $(r=-0.304 ; p=0.018)$, considering the Bonferroni corrected $\mathrm{p}$-value of 0.0125 . Of note, in a multivariate partial correlation, baseline SDNN remained a significant predictor of PSA levels at 6 months from diagnosis, controlling for tumor stage, age, baseline PSA, as well as hormonal treatment and radiotherapy $(\mathrm{r}=-0.434 ; \mathrm{p}=0.004)$ (Fig. 1). Furthermore, baseline RMSSD was a significant predictor of PSA levels at 6 months from diagnosis $(\mathrm{r}=-0.437 ; \mathrm{p}=0.004)$, as well as of PSA levels at 2 years $(r=-0.381 ; p=0.0125)$, independent of all confounders.

Moderation. Using a cut-off of $20 \mathrm{msec}$ for SDNN (29), a significant interaction was found between SDNN and stage $[\mathrm{F}(2,41)=15.262 ; \mathrm{p}<0.001]$ in relation to PSA levels at 6 months. The same significant interaction was observed between the cut-off for RMSSD (also $20 \mathrm{~ms}$ ) and stage $[\mathrm{F}(2,37)=13.194$; $\mathrm{p}<0.001]$ in relation to PSA levels at 6 months. The two interactions were independent of confounders. Following this interaction, we examined the correlation between SDNN and 


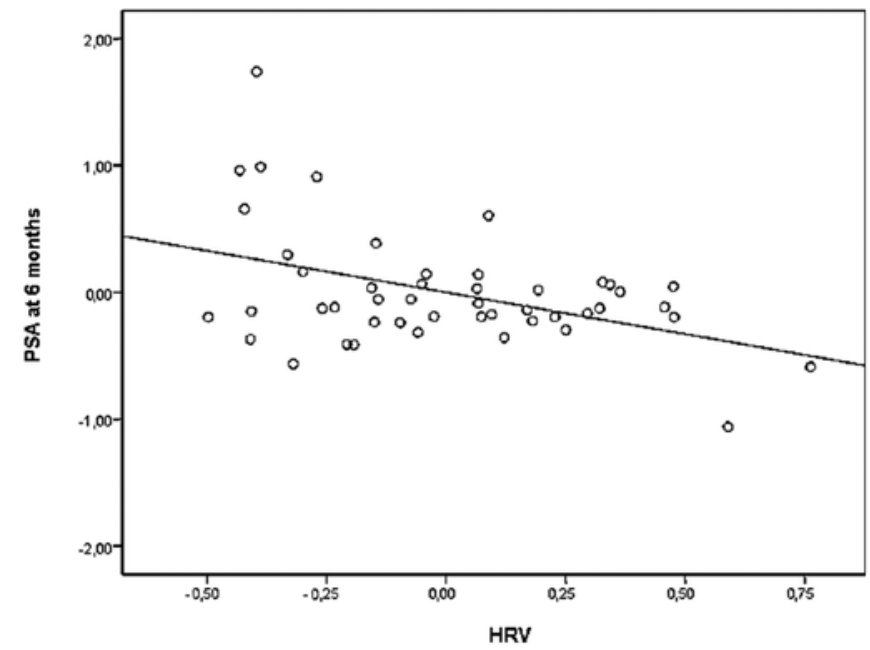

Figure 1. The relationship between HRV $(\log$ SDNN) and $\log$ PSA levels at 6 months. HRV, heart rate variability; SDNN, standard deviation of normal RR intervals; PSA, prostate-specific antigen.

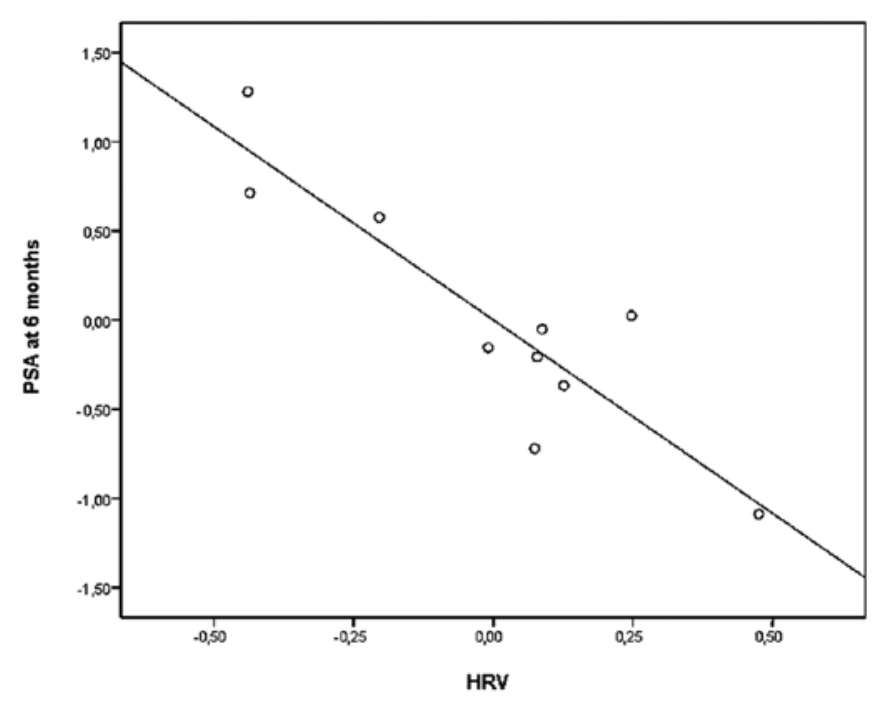

Figure 2. The relationship between HRV (log SDNN) and $\log$ PSA levels at 6 months in stage 4 patients. HRV, heart rate variability; SDNN, standard deviation of normal RR intervals; PSA, prostate-specific antigen.

RMSSD with PSA levels at 6 months, in the 3 stages separately, independent of confounders. In stages 2 and 3, we did not find a significant correlation between either SDNN or RMSSD and PSA levels at 6 months and 2 years ( $p>0.05)$. In stage 4 , however, we found a significant inverse correlation between SDNN and PSA levels at 6 months $(r=-0.895 ; p<0.05)$ (Fig. 2). RMSSD was also significantly inversely correlated with PSA levels at 6 months $(\mathrm{r}=-0.804 ; \mathrm{p}=0.05)$. No significant correlations were found for PSA levels at 2 years in stage 4 ( $p>0.05)$. Age did not significantly interact with SDNN in relation to PSA levels at 6 months $[F(1,43)=0.686 ; p=0.412]$, independent of confounders.

Non-small cell lung cancer. Of the 650 initially reviewed medical charts of NSCLC, only 133 met our inclusion criteria (for the majority of the excluded patients, no ECG was available). The descriptive statistics of the study variables of this
Table II. Descriptive statistics of the NSCLC patient sample.

A. Categorical variables

Variable

Deceased patients

Radiotherapy

Chemotherapy

Surgery

Stage

Stage 1

Stage 2

Stage 3

Stage 4

54.5

B. Continuous variables

\begin{tabular}{lc}
\hline Variable & (Mean \pm SD) \\
\hline Age (years) & $62.2 \pm 10.2$ \\
Heart rate & $82.0 \pm 17.3$ \\
HRV variables & \\
SDNN & $17.0 \pm 14.6$ \\
RMSSD & $19.1 \pm 21.1$ \\
Survival days & $422.6 \pm 391.2$
\end{tabular}

sample are shown in Table II, however they are not log transformed. As shown in Table II, various treatments were used, and the percentages exceeded a $100 \%$ since a multi-modality therapeutic approach was used for several patients.

Due to the lack of normal distributions, the scores of CRP, SDNN, RMSSD and survival time (in days) were log transformed. We then tested the relationship between each measured confounder and OS, and chemotherapy, surgery and stage were found to be significant predictors. Similarly, we tested the covariates for survival time. Among all confounders, radiotherapy, surgery and stage were correlated with survival time. These confounders were considered for their respective analyses. CRP also correlated with OS ( $\mathrm{p}=0.007)$. However, we treated CRP as a variable which may explain (mediate) the relationship between vagal activity and prognosis, thus we did not consider it as a covariate.

Overall survival as outcome. Concerning OS, a multivariate Cox regression analysis was conducted and revealed no relationship between SDNN or RMSSD and OS ( $p=0.357$ and $\mathrm{p}=0.364$, respectively). In this multivariate analysis, only stage and surgery significantly predicted OS $(\mathrm{p}<0.0125)$ (Table III).

We then tested whether there was an interaction between age and SDNN in relation to OS. This interaction was not significant $(B=0.152 ; p=0.703)$ and thus, no subsequent analyses were conducted. Similarly, there was no interaction between stage and SDNN in relation to OS $(\mathrm{B}=-0.068 ; \mathrm{p}=0.737)$. 
Table III. Cox regression analysis for the relationship between cancer stage, treatments and SDNN with OS.

\begin{tabular}{lcccc}
\hline Predictor & B & Sig & R.R. & 95\% CI \\
\hline Stage & 0.621 & 0.000 & 1.861 & $1.484-2.333$ \\
Surgery & -0.948 & 0.002 & 0.388 & $0.215-0.698$ \\
SDNN & -0.176 & 0.357 & 0.838 & $0.576-1.22$ \\
Chemotherapy & -0.667 & 0.014 & 0.513 & $0.302-0.873$
\end{tabular}

SDNN, standard deviation of normal RR intervals; OS, overall survival; $\mathrm{RR}$, relative risk; CI, confidence interval.

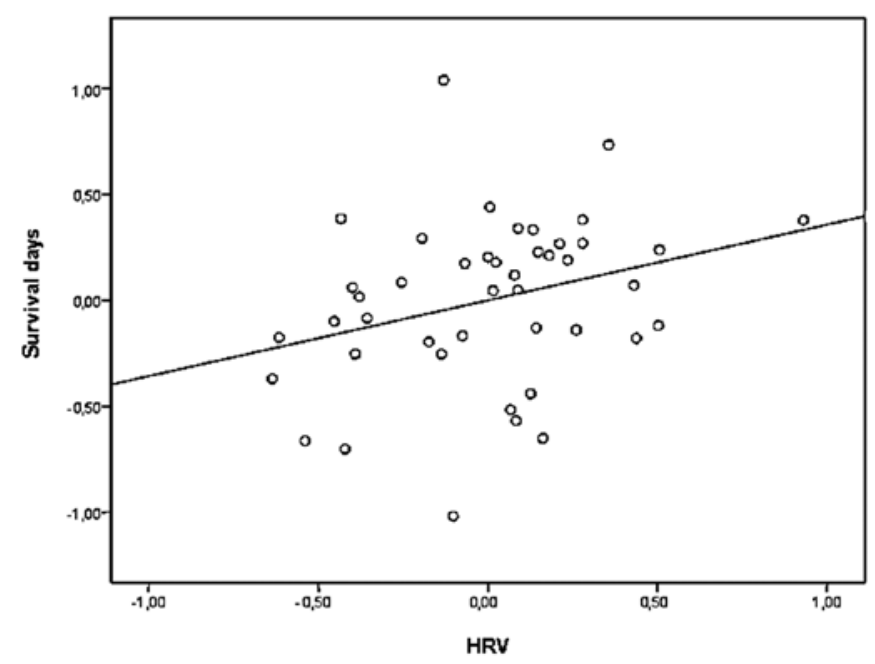

Figure 3. The relationship between HRV (log SDNN) and log survival time in patients $<65$ years old. HRV, heart rate variability; SDNN, standard deviation of normal RR intervals.

We initially hypothesized that CRP may mediate the relation between SDNN and OS. However, since no significant relationship was found between SDNN and OS, as mentioned above, a mediation analysis was not appropriate. However, CRP on its own significantly predicted OS [t(74) $=2.799 ; \mathrm{p}=0.007]$, without controlling for confounders, and this relationship remained significant after controlling for stage, chemotherapy and surgery as well $(\mathrm{B}=0.447 ; \mathrm{p}=0.038)$.

Survival time as outcome. The following analyses were conducted in the deceased NSCLC patients only. In univariate analyses, SDNN and RMSSD did not significantly predict survival time ( $\mathrm{p}=0.24$ and $\mathrm{p}=0.486$, respectively). In a multivariate partial correlation, baseline SDNN and RMSSD were not significantly predictive of survival time, controlling for tumor stage, surgery and radiotherapy $(\mathrm{p}=0.435$ and $\mathrm{p}=0.34$, respectively).

Regarding the moderators, we tested the interaction between stage and SDNN in relation to survival time, however, no interaction was observed $[\mathrm{F}(1,106)=0.005 ; \mathrm{p}=0.943]$. By contrast, age and SDNN significantly interacted in relation to survival time $[F(1,105)=5.577 ; p=0.02]$. In the group under the age of 65 $(\mathrm{N}=73), \mathrm{SDNN}$ (and RMSSD) significantly predicted survival time, independent of confounders $(r=0.278 ; p=0.032$ and $\mathrm{r}=0.282 ; \mathrm{p}=0.029$, respectively) (Fig. 3). By contrast, in the group over the age of 65 , SDNN and RMSSD did not predict survival time $(\mathrm{r}=-0.174 ; \mathrm{p}=0.247$ and $\mathrm{r}=-0.144 ; \mathrm{p}=0.340$, respectively).

Finally, since no significant correlation was found between SDNN and survival time, as mentioned above, a mediation analysis of CRP on the relationship between SDNN and survival time was not appropriate. However, CRP alone significantly predicted survival time $(r=-0.298 ; \mathrm{p}=0.012)$, without controlling for confounders, and this correlation remained significant after controlling for stage, radiotherapy and surgery as well $(r=-0.263 ; p=0.03)$.

\section{Discussion}

The present study tested the potential prognostic role of vagal nerve activity in two different but common types of cancer, i.e., prostate cancer (PC) and non-small cell lung cancer (NSCLC), excluding patients on cardiac medication or with cardiac disease. The effects of vagal nerve activity on clinical outcomes were hypothesized to occur as a result of the known inhibitory roles of this nerve on three mechanisms promoting tumor onset and progression (excessive inflammation, excessive sympathetic activity and oxidative stress) (21). Vagal nerve activity was indexed by heart rate variability (HRV) obtained from a $10 \mathrm{sec}$ ECG at approximately the time of cancer diagnosis. We used a historical prospective design to predict PSA tumor marker levels (in PC) as well as overall survival (OS) and survival time (in NSCLC), by HRV levels measured at approximately the time of diagnosis. In PC, HRV parameters (SDNN and RMSSD) were found to be significantly and inversely correlated with levels of the tumor marker PSA at 6 months and 2 years, independent of confounders including tumor stage and treatment. Moreover, we observed that HRV predicted PSA levels in patients with metastatic prostate cancer, but not in less severe disease stages, emphasizing the moderating role of stage. These findings corroborated with previous studies showing HRV to predict cancer prognosis in several types of cancer (25-29). However, unlike previous studies, the present study used a more rigorous control of confounders, including baseline tumor marker, among the other crucial confounders. Although our strict exclusion criteria reduced the generalizability of our findings, this serves to enhance the ability to interpret our findings and to attribute them to vagal nerve activity, unaffected by interfering variables.

The prognostic role of vagal nerve activity specifically in the metastatic stage of PC remains to be elucidated. It is possible that in the earlier tumor stages, commonly provided treatments such as surgery and radiotherapy are successful in reducing the tumor burden, possibly leaving less of a margin for vagal nerve activity to contribute to the process. By contrast, these treatments may have less impact in later, metastatic stages, where vagal activity could possibly be of more importance. In addition, during the metastatic stage, all three mechanisms considered to underlie the effects of the vagus on tumor burden, i.e., inflammation, oxidative stress and sympathetic activation $(10,13,16)$, may bear a greater role on prognosis. This would then potentially enable greater impact of the vagal nerve on these three processes and on tumor burden in the metastatic stage.

In patients with NSCLC, no correlation was found between SDNN and RMSSD and OS. Furthermore, age and stage did 
not moderate this relationship. Concerning survival time in the deceased patients, no correlation between SDNN and RMSSD and survival time was observed. However, in patients under the age of 65 , higher HRV did significantly predict longer survival time, independent of confounders. The lack of correlation between HRV and prognosis in NSCLC in the elderly may result from the cumulative effects of this cancer with comorbidities (such as cardiac disease) and age on the parasympathetic system. Several respiratory parameters are known to affect HRV, such as respiratory frequency $(36,37)$, tidal volume (37), the time ratio of expiration/inspiration (38), restricted breathing, pulmonary function, functional capacity, and chronic obstructive pulmonary disease (COPD) (39). These respiratory parameters, as well as multiple regulatory systems, are likely to be impaired in older patients with NSCLC (30,40-42).

Another reason why we did not observe a relationship could be explained by the mean SDNN in the NSCLC patients. The NSCLC patients in general had a low SDNN, leading to less variation and hence to a restricted range. Contrasting the two types of cancer we investigated, the mean SDNN of the NSCLC patients was significantly lower than the mean of the PC patients (SDNN, $17 \mathrm{msec}$; SDNN, $31.2 \mathrm{msec}$; $\mathrm{p}<0.005$ ), possibly due to the cancer-induced respiratory problems and the severity of the cancer burden in NSCLC. In addition, younger people in a general population have more variations in their vagal nerve activity and, similarly, more variations were found in this sample of the younger NSCLC patients. This could explain the correlation in the younger subgroup with survival time, as a result of the broader range of HRV parameters and a potentially better regulatory functioning of the autonomic nervous system. Finally, in the NSCLC sample, CRP significantly predicted survival time and OS, as expected, since CRP is a known prognostic parameter in cancer in general, and in NSCLC specifically (43).

The present study had several limitations. First, in both samples, HRV measurements were based on ECGs of $10 \mathrm{sec}$. However, several studies have suggested that HRV indices, obtained from $10 \mathrm{sec}$ ECG, are correlated with 5 and $20 \mathrm{~min}$ ECGs $(34,44)$. Such brief HRV data have also been shown to predict prognosis in cardiac disease (45) and in cancer (29). Another potential limitation could be that the ECG was obtained at approximately the time of diagnosis. Patients had just been informed about their diagnosis and this may have impacted their sympathetic nervous system activity and, consequently, their HRV (46). This may result in an underestimation of the full relationship between HRV and cancer prognosis. Future studies should assess the levels of stress at the moment of ECG measurement. In addition, both sample sizes, although larger than in previous studies, were limited in size for establishing with adequate statistical power the effects of moderators and mediators. Finally, a historical prospective design was used in which a priori control over confounders, such as performance status (including Karnofsky), sampling and ECG measures, was lacking. It is suggested that future studies should replicate these results using a prospective design, with larger samples and longer ECG measurements.

Since our design is correlational, we cannot infer any causal relationship between HRV and tumor burden. It may be that vagal nerve activity is also affected by cancer (De Couck and Gidron, unpublished data). However, two experimental studies in mice showed that vagotomy or capsaicin-induced denervation enhanced cancer metastasis $(47,48)$, demonstrating experimentally the protective effects of the vagus nerve on cancer progression. Furthermore, a recent study found an anti-inflammatory drug that activates the vagus nerve (CNI-1493) to reduce tumor burden in a breast cancer murine model (49). Collectively, these results support the theory that this nerve plays a pivotal homeostatic role whose function may be disease prevention or protection against poor prognosis in certain types of cancer.

\section{Acknowledgements}

The authors thank Professor D. Schoors and Sven D'haese for their contribution to this research. This study was supported by grants from the Reliable Cancer Therapies and the Willy Gepts Foundation provided to Professor Yori Gidron.

\section{References}

1. Global cancer statistics: CA Cancer J Clin 61: 69-90, 2011.

2. Eastham JA: Prostate-specific antigen doubling time as a prognostic marker in prostate cancer. Nat Clin Pract Urol 2: 482-491, 2005.

3. de Bono JS, Logothetis CJ, Molina A, Fizazi K, North S, Chu L, Chi KN, Jones RJ, Goodman OB Jr, Saad F, Staffurth JN, Mainwaring P, Harland S, Flaig TW, Hutson TE, Cheng T, Patterson H, Hainsworth JD, Ryan CJ, Sternberg CN, Ellard SL, Fléchon A, Saleh M, Scholz M, Efstathiou E, Zivi A, Bianchini D, Loriot Y, Chieffo N, Kheoh T, Haqq CM and Scher HI; COU-AA-301 Investigators: Abiraterone and increased survival in metastatic prostate cancer. N Engl J Med 364: 1995-2005, 2011.

4. Kelsey CR, Marks LB, Hollis D, Hubbs JL, Ready NE, D'Amico TA and Boyd JA: Local recurrence after surgery for early stage lung cancer: an 11-year experience with 975 patients. Cancer 115: 5218-5227, 2009

5. Rosell R, Molina MA, Costa C, Simonetti S, Gimenez-Capitan A, Bertran-Alamillo J, Mayo C, Moran T, Mendez P, Cardenal F, Isla D, Provencio M, Cobo M, Insa A, Garcia-Campelo R, Reguart N, Majem M, Viteri S, Carcereny E, Porta R, Massuti B, Queralt C, de Aguirre I, Sanchez JM, Sanchez-Ronco M, Mate JL, Ariza A, Benlloch S, Sanchez JJ, Bivona TG, Sawyers CL and Taron M: Pretreatment EGFR T790M mutation and BRCA1 mRNA expression in erlotinib-treated advanced non-small-cell lung cancer patients with EGFR mutations. Clin Cancer Res 17: 1160-1168, 2011.

6. Gandhi L and Janne PA: Crizotinib for ALK-rearranged non-small cell lung cancer: a new targeted therapy for a new target. Clin Cancer Res 18: 3737-3742, 2012.

7. Brundage MD, Davies D and Mackillop WJ: Prognostic factors in non-small cell lung cancer: a decade of progress. Chest 122: 1037-1057, 2002.

8. Marijon H, Bouyon A, Vignot S and Besse B: Prognostic and predictive factors in lung cancer. Bull Cancer 96: 391-404, 2009.

9. Lee JH, Song EM, Sim YS, Ryu YJ and Chang JH: Forced expiratory volume in one second as a prognostic factor in advanced non-small cell lung cancer. J Thorac Oncol 6: 305-309, 2011.

10. Valko M, Izakovic M, Mazur M, Rhodes CJ and Telser J: Role of oxygen radicals in DNA damage and cancer incidence. Mol Cell Biochem 266: 37-56, 2004.

11. Faux SP, Tai T, Thorne D, Xu Y, Breheny D and Gaca M: The role of oxidative stress in the biological responses of lung epithelial cells to cigarette smoke. Biomarkers 14: 90-96, 2009.

12. Maki A, Kono H, Gupta M, Asakawa M, Suzuki T, Matsuda M, Fujii $\mathrm{H}$ and Rusyn I: Predictive power of biomarkers of oxidative stress and inflammation in patients with hepatitis C virus-associated hepatocellular carcinoma. Ann Surg Oncol 14: 1182-1190, 2007.

13. Pikarsky E, Porat RM, Stein I, Abramovitch R, Amit S, Kasem S, Gutkovich-Pyest E, Urieli-Shoval S, Galun E and Ben-Neriah Y: NF-kappaB functions as a tumour promoter in inflammationassociated cancer. Nature 431: 461-466, 2004. 
14. Voronov E, Shouval DS, Krelin Y, et al: IL-1 is required for tumor invasiveness and angiogenesis. Proc Natl Acad Sci USA 100: 2645-2650, 2003.

15. Mantovani A, Allavena P, Sica A and Balkwill F: Cancer-related inflammation. Nature 454: 436-444, 2008.

16. Entschladen F, Drell TL IV, Lang K, Joseph J and Zaenker KS: Tumour-cell migration, invasion, and metastasis: navigation by neurotransmitters. Lancet Oncol 5: 254-258, 2004.

17. Tracey KJ: Reflex control of immunity. Nat Rev Immunol 9: 418-428, 2009.

18. Ek M, Kurosawa M, Lundeberg T and Ericsson A: Activation of vagal afferents after intravenous injection of interleukin-1beta: role of endogenous prostaglandins. J Neurosci 18: 9471-9479, 1998.

19. Pavithran P, Nandeesha H, Sathiyapriya V, Bobby Z and Madanmohan T: Short-term heart variability and oxidative stress in newly diagnosed essential hypertension. Clin Exp Hypertens 30: 486-496, 2009.

20. Vlcek M, Radikova Z, Penesova A, et al: Heart rate variability and catecholamines during hypoglycemia and orthostasis. Auton Neurosci 143: 53-57, 2008.

21. De Couck M, Mravec B and Gidron Y: You may need the vagus nerve to understand pathophysiology and to treat diseases. Clin Sci 122: 323-328, 2012.

22. Gidron Y, Perry H and Glennie M: Does the vagus nerve inform the brain about preclinical tumours and modulate them? Lancet Oncol 6: 245-248, 2005.

23. Task Force of the European Society of Cardiology and the North American Society of Pacing and Electrophysiology: Heart rate variability: standards of measurement, physiological interpretation and clinical use. Circulation 93: 1043-1065, 1996.

24. Kuo TB, Lai C J, Huang YT and Yang CC: Regression analysis between heart rate variability and baroreflex-related vagus nerve activity in rats. J Cardiovasc Electrophysiol 16: 864-869, 2005.

25. Hoffmann J, Grimm W, Menz V, Wied M, Sprenger A, Arnold R and Maisch B: Prognostic value of heart rate variability analysis in patients with carcinoid syndrome. Digestion 63: 35-42, 2001.

26. Fadul N, Strasser F, Palmer LJ, Yusuf SW, Guo Y, Li Z, Allo J and Bruera E: The association between autonomic dysfunction and survival in male patients with advanced cancer: a preliminary report. J Pain Symptom Manage 39: 283-290, 2010.

27. Chiang JK, Koo M, Kuo TB and Fu CH: Association between cardiovascular autonomic functions and time to death in patients with terminal hepatocellular carcinoma. J Pain Symptom Manage 39: 673-679, 2010.

28. Kim do H, Kim JA, Choi YS, Kim SH, Lee JY and Kim YE: Heart rate variability and length of survival in hospice cancer patients. J Korean Med Sci 25: 1140-1145, 2010.

29. Mouton C, Ronson A, Razavi D, Delhaye F, Kupper N, Paesmans M, Moreau M, Nogaret JM, Hendlisz A and Gidron Y: The relationship between heart rate variability and time-course of carcinoembryonic antigen in colorectal cancer. Auton Neurosci 166: 96-99, 2012.

30. Crimmins EM, Johnston M, Hayward M and Seeman T: Age differences in allostatic load: an index of physiological dysregulation. Exp Gerontol 38: 731-734, 2003.

31. Pfleger CC, Flachs EM and Koch-Henriksen N: Social consequences of multiple sclerosis: clinical and demographic predictors - a historical prospective cohort study. Eur J Neurol 17: 1346-1351, 2010.

32. Lerner-Geva L, Keinan-Boker L, Blumstein T, Boyko V, Olmar L, Mashiach S, Rabinovici J, Potashnik G, Lunenfeld E, Schenker JG, Shushan A, Fishman A, Cohen I, Vagman I and Lunenfeld B: Infertility, ovulation induction treatments and the incidence of breast cancer - a historical prospective cohort of Israeli women. Breast Cancer Res Treat 100: 201-212, 2006.
33. Thong T, Li K, McNames J, Aboy M and Goldstein B: Accuracy of ultra-short heart rate variability measures. Engineering in Medicine and Biology Society. Proc 25th Annual Int Conference of the IEEE 3: 2424-2427, 2003.

34. Nussinovitch U, Elishkevitz KP, Katz K, Nussinovitch M, Segev S, Volovitz B and Nussinovitch N: Reliability of ultrashort ECG indices for heart rate variability. Ann Noninvasive Electrocardiol 16: 117-122, 2011.

35. Hodgart E and Macfarlane PW: 10 second heart rate variability. Comp Cardiol 31: 217-220, 2004.

36. Schipke JD, Pelzer M and Arnold G: Effect of respiration rate on short-term heart rate variability. J Clin Basic Cardiol 2: 92-94, 1999.

37. Poyhonen M, Syvaoja S, Hartikainen J, Ruokonen E and Takala J: The effect of carbon dioxide, respiratory rate and tidal volume on human heart rate variability. Acta Anaesthesiol Scand 48: 93-101, 2004

38. Strauss-Blasche G, Moser M, Voica M, McLeod DR, Klammer N and Marktl W: Relative timing of inspiration and expiration affects respiratory sinus arrhythmia. Clin Exp Pharmacol Physiol 27: 601-606, 2000.

39. Brown JK, Cooley ME, Chernecky C and Sarna L: A symptom cluster and sentinel symptom experienced by women with lung cancer. Oncol Nurs Forum 38: E425-E435, 2011.

40. Johannessen A, Lehmann S, Omenaas ER, Eide GE, Bakke PS and Gulsvik A: Post-bronchodilator spirometry reference values in adults and implications for disease management. Am J Respir Crit Care Med 173: 1316-1325, 2006.

41. Katona PG and Jih F: Respiratory sinus arrhythmia: noninvasive measure of parasympathetic cardiac control. J Appl Physiol 39: 801-805, 1975.

42. Cassart M, Pettiaux N, Gavenois PA, Pavia M and Estenne M: Effect of chronic hyperinflation on diaphragm length and surface area. Am J Respir Crit Care Med 156: 504-508, 1997.

43. Alifano M,Falcoz PE, Seegers V, Roche N, Schussler O, Younes M, Antonacci F, Forgez P, Dechartres A, Massard G, Damotte D and Régnard JF: Preresection serum C-reactive protein measurement and survival among patients with resectable non-small cell lung cancer. J Thorac Cardiovasc Surg 142: 1161-1167, 2011.

44. Hamilton RM, McKechnie PS and Macfarlane PW: Can cardiac vagal tone be estimated from the 10-second ECG? Int J Cardiol 95: 109-115, 2004.

45. Dekker JM, Schouten EG, Klootwijk P, Pool J, Swenne CA and Kromhout D: Heart rate variability from short electrocardiographic recordings predicts mortality from all causes in middle-aged and elderly men. The Zutphen Study. Am J Epidemiol 145: 899-908, 1997.

46. O'Donnell K, Brydon L, Wright CE and Steptoe A: Self-esteem levels and cardiovascular and inflammatory responses to acute stress. Brain Behav Immun 22: 1241-1247, 2008.

47. Erin N, Akdas Barkan G, Harms JF and Clawson GA: Vagotomy enhances experimental metastases of 4THMpc breast cancer cells and alters substance P level. Regul Pept 151: 35-42, 2008.

48. Erin N, Boyer PJ, Bonneau RH, Clawson GA and Welch DR: Capsaicin-mediated denervation of sensory neurons promotes mammary tumor metastasis to lung and heart. Anticancer Res 24: 1003-1009, 2004.

49. Erin N, Duymus O, Oztürk S and Demir N: Activation of vagus nerve by semapimod alters substance $\mathrm{P}$ levels and decreases breast cancer metastasis. Regul Pept 179: 101-108, 2012. 